\title{
The Biomechanical Effects of Limited Lateral Retinacular and Capsular Release on Lateral Patellar Translation at Various Flexion Angles in Cadaveric Specimens
}

\author{
Jourdan M. Cancienne, M.D., David R. Christian, M.D., Michael L. Redondo, M.D., \\ Hailey P. Huddleston, B.S., Elizbeth F. Shewman, M.S., Jack Farr, M.D., \\ Brian J. Cole, M.D., M.B.A., and Adam B. Yanke, M.D., Ph.D.
}

\begin{abstract}
Purpose: To determine the biomechanical effect of limited lateral retinacular and capsular release on lateral patellar translation as a function of constant force at various knee flexion angles. Methods: Six pairs of bilateral cadaveric knee specimens (12 knees) were obtained from a tissue bank, dissected, and potted in a perfect lateral position based on fluoroscopy. A direct lateral force was applied to the patella through an eye screw in the midpoint of the lateral patella, and each knee underwent testing in the intact state and after lateral retinacular and capsular release. All knees were tested at $0^{\circ}, 10^{\circ}, 20^{\circ}, 30^{\circ}, 45^{\circ}, 60^{\circ}$, and $90^{\circ}$ of flexion using a custom-machined jig on a materials testing system with a $20-\mathrm{N}$ lateral force applied to the patella. Patellar displacement was recorded and compared for each specimen. Results: Lateral displacement was significantly greater at all degrees of flexion for the lateral-release specimens than for an intact lateral retinaculum $(P<.05)$. Compared with intact specimens, lateral-release specimens experienced $30 \%$ more translation at $0^{\circ}$ of flexion and between $6 \%$ and $9 \%$ more lateral translation at $10^{\circ}$ to $90^{\circ}$ of flexion. Conclusions: Lateral retinacular and capsular release results in significantly increased lateral patellar translation at all flexion angles compared with intact specimens. This finding suggests that the lateral retinaculum may function as a significant restraint to lateral translation even with intact medial soft-tissue restraints. Clinical Relevance: Arthroscopic and open limited lateral retinacular releases should be performed with extreme caution when treating lateral patellar instability given the lateral retinaculum's apparent role as a secondary restraint.
\end{abstract}

From the Department of Orthopaedics, Rush University Medical Center (J.M.C., H.P.H., E.F.S., B.J.C., A.B.Y.), Chicago, Illinois; McGaw Medical Center at Northwestern University (D.R.C.), Chicago, Illinois; University of Illinois at Chicago (M.L.R.), Chicago, Illinois; and OrthoIndy (J.F.), Greenwood, Indiana, U.S.A.

The authors report the following potential conflicts of interest or sources of funding: J.F. receives research support from Active Implants, Fidia, Novartis, and Zimmer Biomet; is on the editorial or governing board of the American Journal of Orthopaedics and Cartilage; receives IP royalties from and is a paid presenter or speaker for Arthrex; is a paid consultant for Cartiheal, Exactech, Regentis, Samumed, and Zipline; receives stock or stock options from MedShape and Ortho Regenerative Tech; is a paid consultant and paid presenter or speaker for and receives research support from Moximed; is a paid consultant and paid presenter or speaker for and receives IP royalties from Organogenesis; receives publishing royalties and financial or material support from Springer and Thieme Medical Publishers; and is a paid presenter or speaker for Vericel. B.J.C. receives research support from Aesculap/B.Braun and National Institutes of Health (NIAMS and NICHD); is on the editorial or governing board of the American Journal of Orthopedics, American Journal of Sports Medicine, Cartilage, Journal of Shoulder and Elbow Surgery, and Journal of the American Academy of Orthopaedic Surgeons; receives IP royalties and research support from and is a paid consultant for Arthrex; is a board or committee member of the Arthroscopy Association of North America and International Cartilage Repair Society; receives other financial or material support from Athletico, JRF Ortho, and Smith $\theta$ Nephew; receives IP royalties from Elsevier Publishing; receives publishing royalties and financial or material support from Operative Techniques in Sports Medicine; receives stock or stock options from Ossio; and is a paid consultant for and receives research support and stock or stock options from Regentis. A.B.Y. receives grant support from the Arthroscopy Association of North America; is a paid consultant for JRF Ortho; and receives research funding from Arthrex and Organogenesis. Full ICMJE author disclosure forms are available for this article online, as supplementary material.

Received April 20, 2019; accepted September 8, 2019.

Address correspondence to Adam B. Yanke, M.D., Ph.D., Department of Orthopaedics, Rush University Medical Center, 1611 W Harrison St, Ste 300, Chicago,IL 60612,U.S.A.E-mail: adam.yanke@rushortho.com

(C) 2019 Published by Elsevier on behalf of the Arthroscopy Association of North America. This is an open access article under the CC BY-NC-ND license (http://creativecommons.org/licenses/by-nc-nd/4.0/).

2666-061X/19520

https://doi.org/10.1016/j.asmr.2019.09.002 
$\mathbf{P}$ atellofemoral joint stability and patellar tracking rely on several physiological and anatomic variables, including the extensor mechanism, retinacular restraints, articular geometry of the patella and trochlea, ligamentous elasticity, and overall limb alignment. ${ }^{1}$ The relative contribution of each of these factors to patellar instability is currently unclear. Thus, the surgical correction of patellofemoral instability is complex, with multiple procedures aimed at correcting each factor thought to be contributing to instability. Lateral retinacular and capsular release is one such procedure that was historically used to treat lateral patellar instability..$^{2-4}$ Although isolated lateral retinacular and capsular releases were commonly performed for various patellofemoral pathologies, the indications for isolated release have narrowed. ${ }^{2,5-9}$ Whereas isolated lateral release is not recommended by most patellofemoral experts, it is still performed for lateral patellar instability by some surgeons. ${ }^{10}$ This procedure is more commonly used in combination with medial patellofemoral ligament reconstruction. ${ }^{6,11}$ Despite studies reporting satisfactory results after isolated lateral release for the treatment of patellofemoral instability, other studies have shown an increase in not only medial instability but also lateral instability after lateral retinacular release. ${ }^{12,13}$ Although several biomechanical studies have focused on the increase in medial patellar instability after lateral release, few have investigated the contribution of the lateral retinaculum and capsule to lateral patellar instability. ${ }^{14-16}$ In those studies that have evaluated the role of the lateral structures in lateral patellar instability, patellar force-displacement behavior was described as the force required to laterally translate the patella a minimum distance. ${ }^{14-16}$ Conversely, patellar displacement as a function of constant force has been less well examined biomechanically, and this may be more clinically relevant. This is because with a set amount of force applied, inherent joint laxity may have a decreased overall effect on results. Along with this, prior biomechanical evaluations have used a larger lateral release, not reflective of a more modern understanding of the lateral patellar anatomy..$^{14-16}$

The purpose of the study was to determine the biomechanical effect of limited lateral retinacular and capsular release on lateral patellar translation as a function of constant force at various knee flexion angles. We hypothesized that a lateral retinacular and capsular release would increase lateral displacement at all degrees of flexion compared with specimens with an intact retinaculum and capsule, with the largest difference in full extension.

\section{Methods}

This study was granted exemption by the institutional review board because it did not involve human subjects. Six pairs of fresh-frozen bilateral cadaveric knees
(12 knees total) were obtained from a tissue bank (Medcure, Portland, OR). The mean age was 52.2 years (range, 39-63 years). The exclusion criteria for specimens obtained included age older than 65 years and a history of any of the following: lower-extremity surgery, knee disease, lower-extremity trauma, cancer, inflammatory arthritis, any connective disorder such as Ehlers-Danlos syndrome, and a bedridden condition. The knees were sealed and stored at $-20^{\circ} \mathrm{C}$. When scheduled for testing, they were thawed to room temperature 24 hours prior to examination.

\section{Specimen Preparation}

The skin, subcutaneous tissue, fascia, and muscle overlying the proximal aspect of the femur and distal aspect of the tibia were removed circumferentially to allow for potting, with care taken to preserve the deep fascia, quadriceps tendon and distal musculature, patellar tendon, retinacula, iliotibial band, and knee joint. By use of fluoroscopy, the proximal femur and distal tibia of each knee were potted in the position of a perfect lateral radiograph to align the posterior aspects of the femoral condyles to ensure that a completely lateral force would be applied to the patella during testing. A Kirschner wire was then used to secure the distal femur in a polyvinyl chloride pipe at the correct angle prior to the addition of polymethyl methacrylate. The quadriceps tendon was identified, and Krackow sutures were placed in the tendon using No. 1 FiberWire (Arthrex, Naples, FL) to allow for quadriceps loading during testing. Diagnostic arthroscopy was then performed through a standard anterolateral portal to

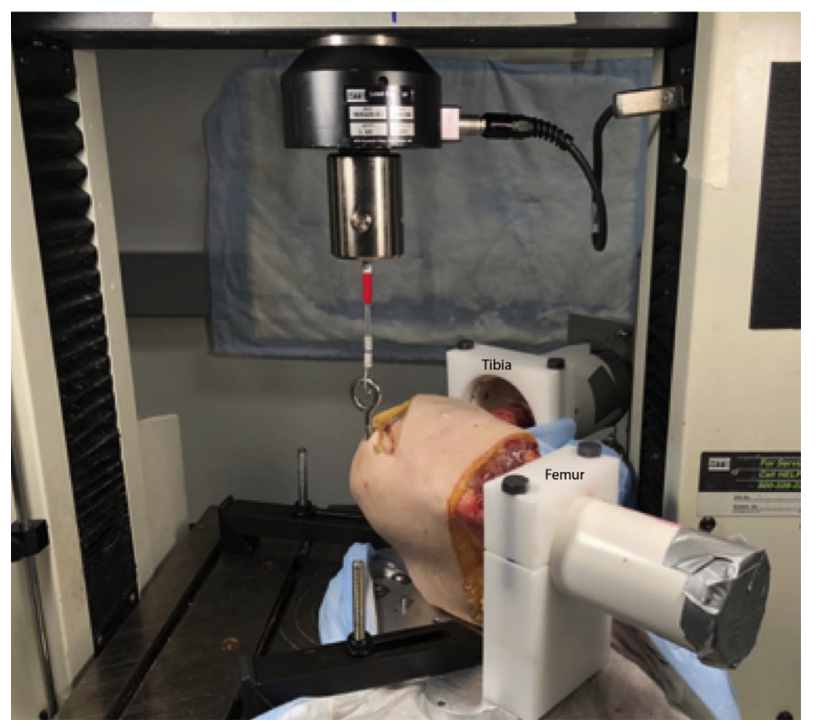

Fig 1. Experimental setup. The tibia and femur were potted using polymethyl methacrylate and loaded into the custom jig as shown. An eye screw was placed perpendicular to the lateral aspect of the midpoint of the patella and connected to the materials testing system via a cable. 
evaluate for ligamentous integrity, normal trochlear morphology, and evidence of osteoarthritis. The biomechanical setup of the specimens was performed using components of prior published protocols from Desio et al. ${ }^{16}$ and Nomura et al. ${ }^{17}$ (Fig 1). A caliper was used to measure the total length of the patella from proximal to distal, and a $6.3-\mathrm{mm} \times 63.5-\mathrm{mm}$ eye screw was placed perpendicular to the sagittal plane in the lateral aspect of the patella at the midpoint as measured with a caliper through a $0.5-\mathrm{cm}$ skin incision. Rotation and tilt of the patella were not constrained. The potted specimens were then placed in a lateral position in a custom-machined jig that allowed range of motion from $0^{\circ}$ to $90^{\circ}$ of flexion in $5^{\circ}$ increments.

\section{Specimen Testing}

All 12 specimens were first tested in the intact state. Once a specimen was secured in the custom-machined jig, a $1-\mathrm{kg}$ load was applied to the quadriceps tendon by attaching a dumbbell to the FiberWire implanted in the quadriceps tendon. The knee was then positioned so that the patella was located directly below the load cell of the materials testing system (Insight 5; MTS Systems, Eden Prairie, MN) (Fig 1). The 1-kg load applied to the quadriceps during testing was chosen because this has been used in several published biomechanical studies modeling patellar instability in the literature (Desio et al., ${ }^{16}$ 1998; Hautamaa et al., ${ }^{18}$ 1998; and Nomura et al., ${ }^{17}$ 2000). The eye screw fixed in the patella was secured to the load cell by a steel cable. A tensile force was applied at $1.0 \mathrm{~mm} / \mathrm{s}$ until a $20-\mathrm{N}$ direct lateral force was applied to the patella. A $20-\mathrm{N}$ force was chosen based on prior published peer work, in which loads were measured when the patella was laterally displaced to either 1 or $1.90 \mathrm{~cm}$, which is also clinically relevant displacement. ${ }^{12,19,20}$ Before data collection in our study, pilot work using 2 distinct specimens repeatedly resulted in patellar displacements in a similar range when the patella was loaded at a constant rate between 0.1 and $20 \mathrm{~N}$. This was generally true for all flexion angles and degrees of dissection. In addition, the $20-\mathrm{N}$ load was sufficient to result in a load-displacement curve outside of the toe-in region. Load and lateral translation of the patella were recorded at $40 \mathrm{~Hz}$, and the final displacement at $20 \mathrm{~N}$ was reported. Testing was performed 3 times at $0^{\circ}, 10^{\circ}, 20^{\circ}, 30^{\circ}, 45^{\circ}, 60^{\circ}$, and $90^{\circ}$ of flexion, and the averages were recorded. Our test machine (Insight 5) had a position resolution of 0.001 $\mathrm{mm}$ and position accuracy of $0.01 \mathrm{~mm}$. In addition, 3 trials for each test were averaged to determine the end displacement. After completion of testing in the intact state, an open lateral retinacular and capsular release was performed by the senior author (A.B.Y.) and the conditions of testing were repeated for the lateral release state. Similarly to a limited lateral patellar arthrotomy, the lateral retinaculum was completely transected from the superior pole to the inferior pole of the patella. The extent of the limited release is shown in Figure 2 relative to the traditional open lateral release.

\section{Statistical Analysis}

Statistical analysis was conducted using SPSS software (version 24; IBM, Armonk, NY). Lateral patellar translation was not normally distributed as assessed with the Shapiro-Wilk test of residuals. In addition, the data did not meet the distributional assumption of the Wilcoxon signed rank test procedure. Therefore, an exact sign test was used to determine the effect of lateral release on lateral displacement at each measured flexion angle $\left(0^{\circ}\right.$, $10^{\circ}, 20^{\circ}, 30^{\circ}, 45^{\circ}, 60^{\circ}$, and $90^{\circ}$ ). Because of the presence of outliers and lack of normality, the Friedman test was used to analyze the effect of flexion on the ratio of lateral patellar displacement in lateral-release knees to that in intact knees. Pair-wise comparisons were used to examine significant differences between each degree of flexion. Significance was set at $P<.05$.

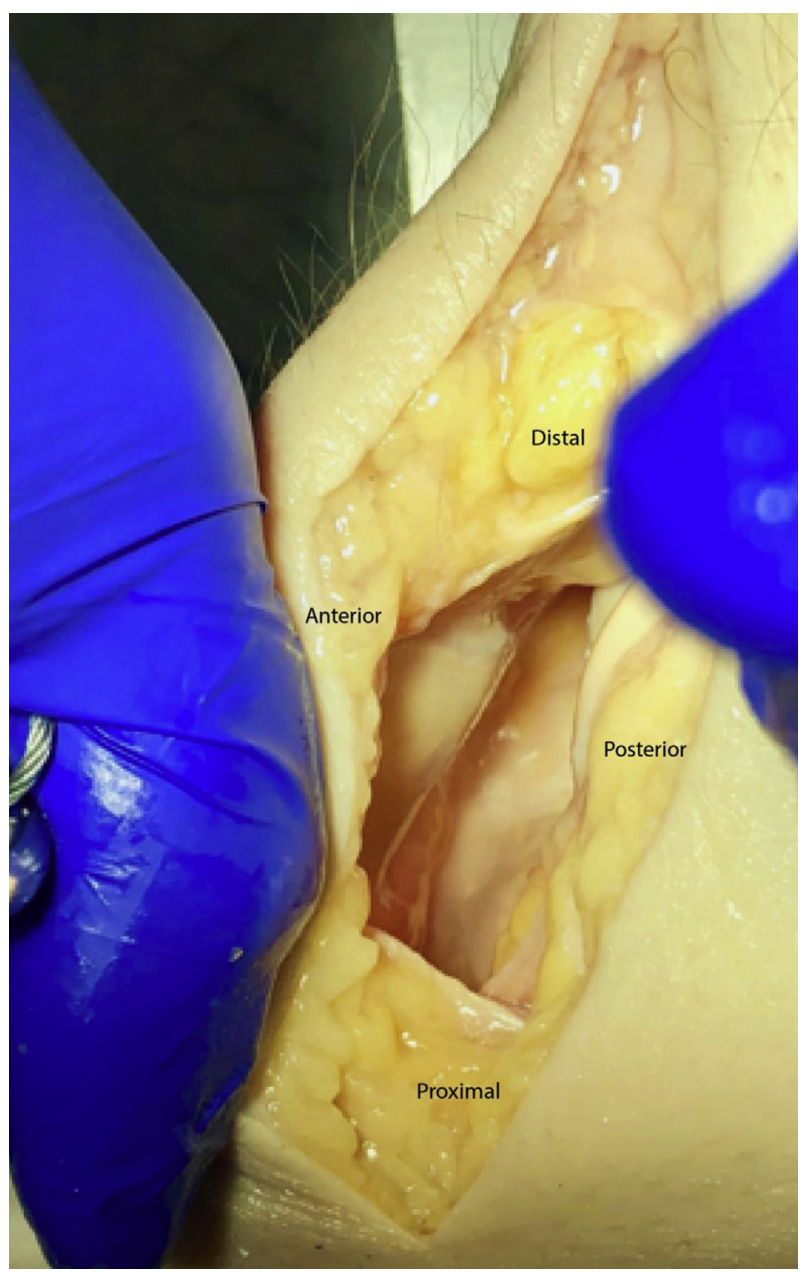

Fig 2. Extent of lateral release procedure. The lateral retinaculum was transected from the superior pole to the inferior pole of the patella. 
Table 1. Mean and Median Displacement of Intact and Lateral-Release Specimens as Function of Knee Flexion

\begin{tabular}{|c|c|c|c|c|c|}
\hline \multirow[b]{3}{*}{ Flexion, $^{\circ}$} & \multicolumn{4}{|c|}{ Displacement, mm } & \multirow[b]{3}{*}{ Difference in Median Displacement, $\mathrm{mm}$} \\
\hline & \multicolumn{2}{|c|}{ Intact Specimens } & \multicolumn{2}{|c|}{ Lateral-Release Specimens } & \\
\hline & Mean $(95 \% \mathrm{CI})$ & Median & Mean, mm (95\% CI) & Median & \\
\hline 10 & $11.69(10.60-12.78)$ & 11.48 & $12.52(11.33-13.71)$ & 12.15 & 0.67 \\
\hline 20 & $11.01(9.87-12.16)$ & 11.06 & $11.90(10.67-13.11)$ & 11.76 & 0.70 \\
\hline 30 & $10.48(9.44-11.52)$ & 10.49 & $11.33(10.14-12.52)$ & 11.04 & 0.55 \\
\hline 90 & $8.74(7.87-9.60)$ & 8.80 & $9.40(8.49-10.30)$ & 8.45 & 0.35 \\
\hline
\end{tabular}

CI, confidence interval.

\section{Results}

Diagnostic arthroscopy of all specimens revealed ligamentously intact specimens without evidence of prior surgery or significant patellofemoral pathology, osteoarthritis, or dysplasia. Mean and median displacement of intact and lateral-release specimens as a function of knee flexion is displayed in Table 1 , with mean values presented in Figure 3. The largest mean lateral displacement was seen at $10^{\circ}$ of knee flexion, and the least, at $90^{\circ}$ of flexion. A significant difference was found between the lateral-release and intact cohorts at each measured degree of flexion $(P<.0005)$. The greatest difference in median values was seen at $0^{\circ}$ of flexion (median difference, $3.95 \mathrm{~mm} ; P<.0005 ; 35$ of 36 samples had a positive difference). The smallest difference was seen at $90^{\circ}$ (median difference, $0.35 \mathrm{~mm} ; P<.0005 ; 32$ of 36 samples had a positive difference).

The ratio of displacement in lateral-release knees to that in intact knees was then compared across degrees of flexion (Fig 4). A significant effect of flexion on patellar lateral displacement was observed $(P<.0005)$ (Table 2$)$. Pair-wise comparisons showed that the displacement in full extension was significantly different from that at all other angles of flexion $\left(P<.0005\right.$ for $0^{\circ}$ vs $10^{\circ}, 0^{\circ}$ vs $20^{\circ}$, $0^{\circ}$ vs $30^{\circ}, 0^{\circ}$ vs $40^{\circ}, 0^{\circ}$ vs $45^{\circ}, 0^{\circ}$ vs $60^{\circ}$, and $0^{\circ}$ vs $90^{\circ}$ ). However, no additional significant differences were seen when comparing displacement ratios at other degrees of flexion. Similarly, the percentage increase, with respect to the intact state, in lateral translation of each specimen after lateral retinacular and capsular release was highest in full extension (Fig 5, Table 3). Again, lateral displacement at $0^{\circ}$ was significantly different from all other flexion angles.

\section{Discussion}

The main findings of this study are that limited lateral retinacular and capsular release results in significantly increased lateral patellar translation at all flexion angles

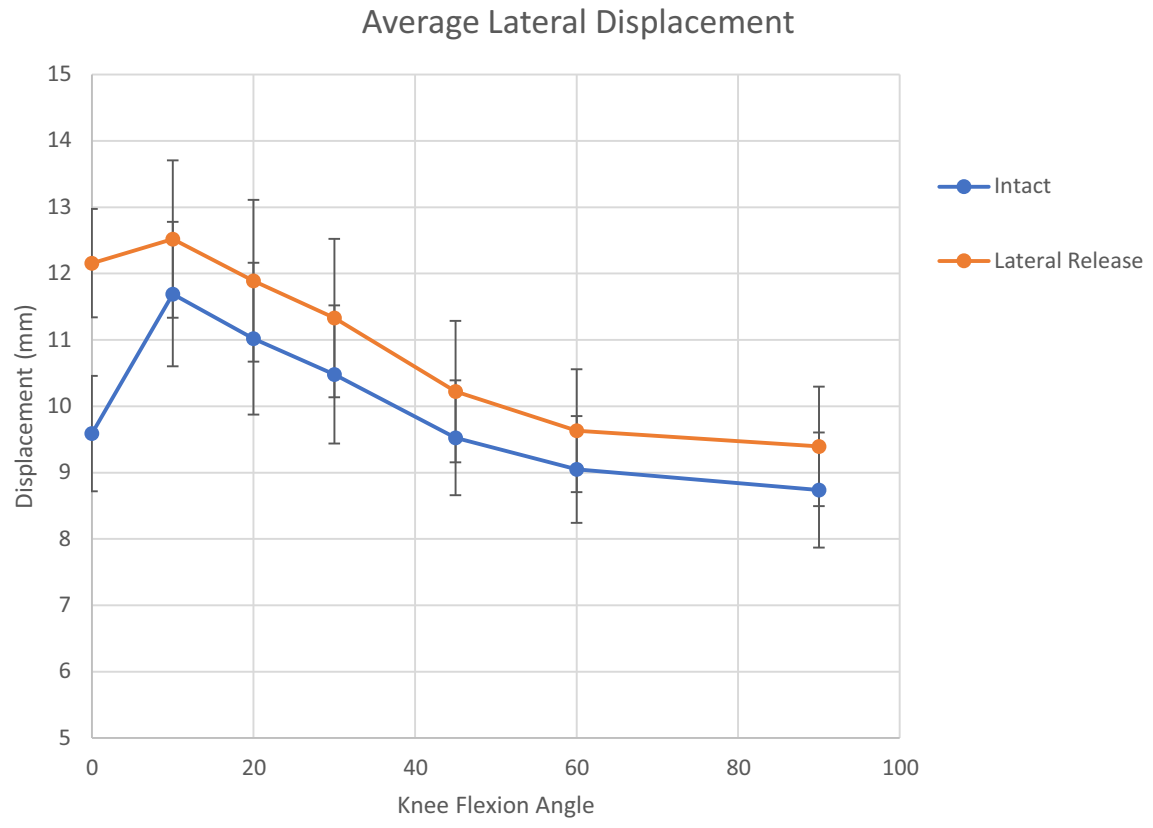

Fig 3. Mean lateral patellar displacement of intact state (blue) and after lateral release (orange) at various degrees of knee flexion. 
Fig 4. Displacement after lateral release (orange) relative to intact state (blue) at various degrees of flexion.

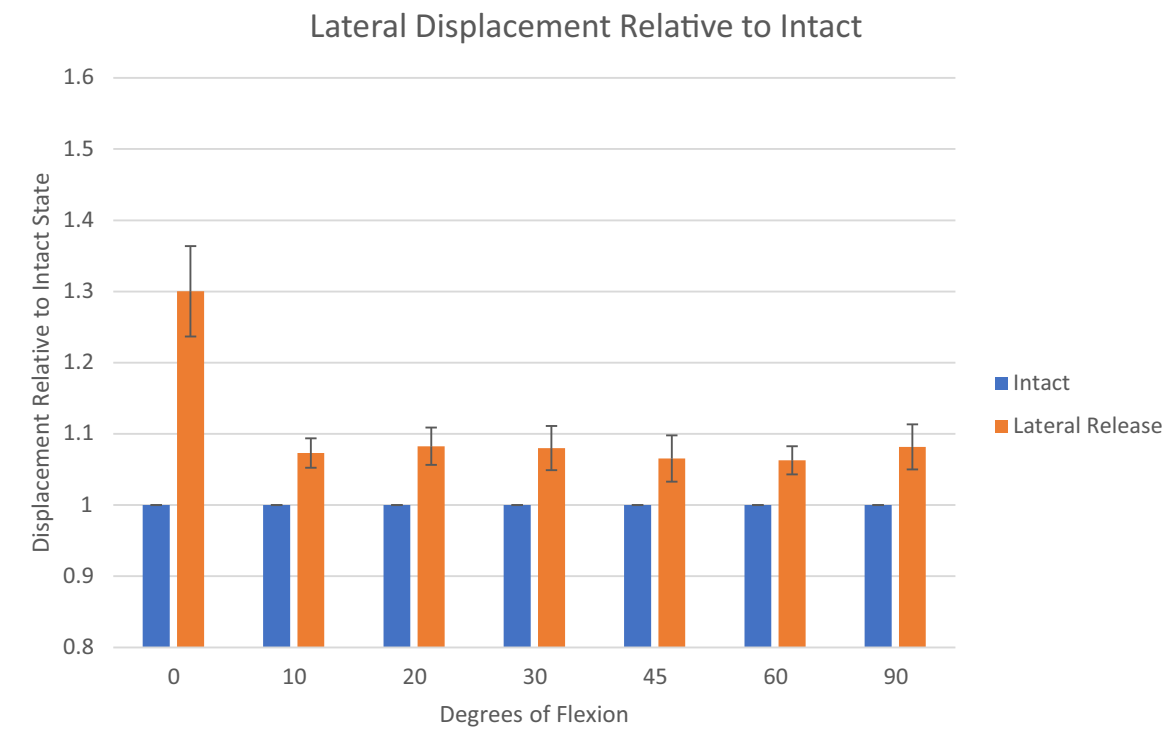

Lateral Displacement Relative to Intact compared with intact specimens. Furthermore, the largest increase in lateral translation in the lateralrelease specimens was seen in full extension. This finding suggests that the lateral retinaculum and capsule function as a significant secondary restraint to lateral translation and that this release might reduce lateral patellar stability.

Three prior biomechanical studies have evaluated the contribution of lateral structures to lateral patellar stability. ${ }^{14-16}$ When one is evaluating these studies, it is important to consider the methods by which the lateral structures were released, as well as how they compare to our modern understanding of the lateral patellofemoral ligament (LPFL) location and more contemporary arthroscopic lateral release techniques. For example, recent anatomic evidence has shown that the insertion of the LPFL on the patella is reliably found to comprise around $45 \%$ of the lateral articular surface in the middle third of the lateral patella. ${ }^{21}$ In our study, we aimed to perform a limited and targeted release of the LPFL to examine how this impacts patellofemoral stability. Merican et al. ${ }^{15}$ evaluated the force required to displace the patella $10 \mathrm{~mm}$ medially and laterally in 9 cadaveric knees with progressive lateral releases. These lateral releases included proximal, middle, distal, and capsular releases. The middle release in their study is most similar to the release performed in our study. Merican et al. noted a significantly greater decrease in medial stability compared with lateral stability with progressive releases. Specifically, when they performed the middle release, lateral stability decreased by an average of $5 \% \pm 5 \%$ of the stability of the intact knee across $30^{\circ}$ to $90^{\circ}$ of flexion. Furthermore, with the addition of the capsular release, they noted no additional instability compared with the middle retinacular release. Our study shows increased lateral instability across all degrees of flexion compared with the intact state. Similarly, Christoforakis et al. ${ }^{14}$ performed a cadaveric study to measure patellar force-displacement behavior at various degrees of flexion by measuring the force required to laterally displace the patella $10 \mathrm{~mm}$ laterally in 7 cadavers. They reported that the mean force required to displace the patella $10 \mathrm{~mm}$ laterally was significantly reduced by an average of $16 \%$ to $19 \%$ from $0^{\circ}$ to $20^{\circ}$ of flexion with an isolated lateral retinacular release. In addition, there was a reduction by $16 \%$ in the force needed to displace the patella $10 \mathrm{~mm}$ laterally at $0^{\circ}$ and $10^{\circ}$ and a reduction by $19 \%$ at $20^{\circ}$. Furthermore, Christoforakis et al. reported that although it was statistically insignificant, the average force required to displace the patella laterally at all other flexion angles was reduced compared with the intact state: $14 \%$ at $30^{\circ}, 7 \%$ at $45^{\circ}$, and $10 \%$ at $60^{\circ}$. However, when one is interpreting the results of their study, it is important to note that the lateral release was conducted using an outside-inward technique from 2 $\mathrm{cm}$ proximal to the upper pole of the patella down to the Gerdy tubercle. This is a much larger and

Table 2. Ratio of Displacement in Lateral-Release Specimens Compared With Intact Specimens as Function of Knee Flexion

\begin{tabular}{lc}
\hline Flexion, $^{\circ}$ & $\begin{array}{c}\text { Mean Ratio of } \\
\text { Displacement } \\
(95 \% \mathrm{CI})\end{array}$ \\
\hline $0^{*}$ & $1.30(1.24-1.36)$ \\
10 & $1.07(1.05-1.09)$ \\
20 & $1.08(1.06-1.11)$ \\
30 & $1.08(1.05-1.11)$ \\
45 & $1.07(1.03-1.10)$ \\
60 & $1.06(1.04-1.08)$ \\
90 & $1.08(1.05-1.11)$ \\
\hline CI, confidence interval. & \\
${ }^{*}$ Significant finding $(P<0.05)$. &
\end{tabular}




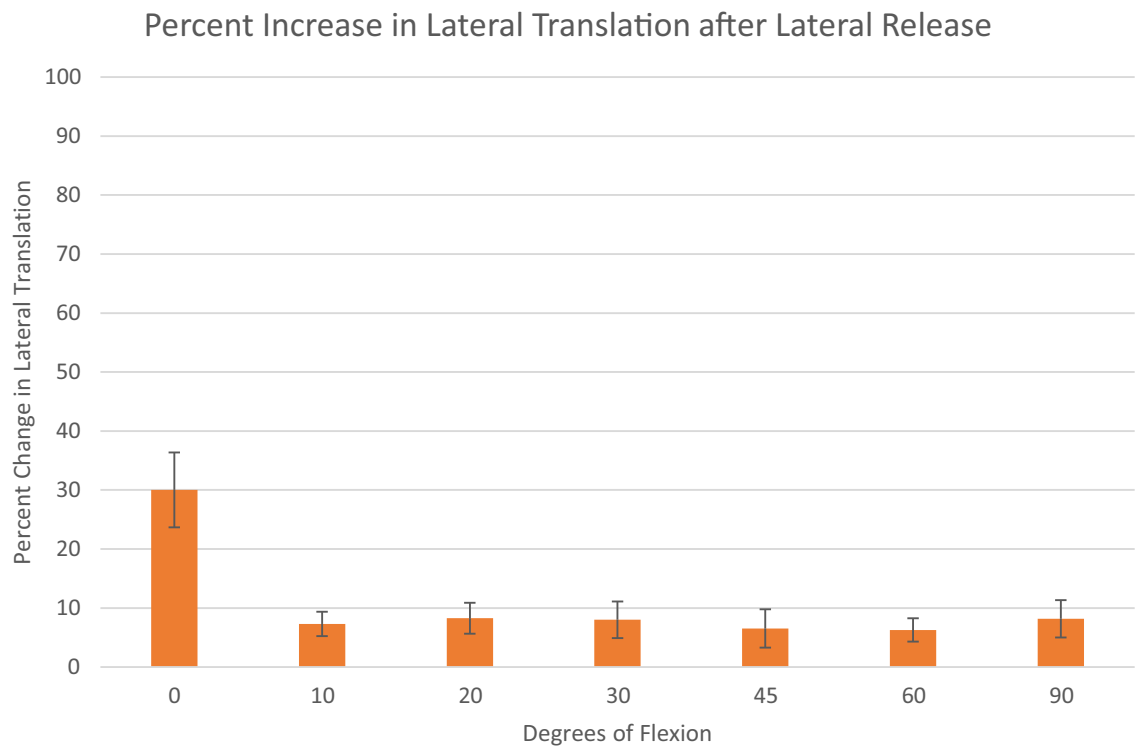

Fig 5. Percentage of lateral patellar translation after lateral retinacular and capsular release relative to intact state at various degrees of flexion. indiscriminate release than that performed in our study, and it does not target the LPFL; thus, it may be clinically less relevant. Finally, Desio et al. ${ }^{16}$ studied the contribution of the lateral structures to lateral patellar translation at $20^{\circ}$ of flexion. They concluded that the medial patellofemoral ligament contributed $60 \%$ of the total restraining force and the lateral retinaculum contributed $10 \%$ (range, 6\%-16\%). Similarly to the aforementioned study by Desio et al., the lateral release was performed from the level of the tibial tubercle to the interval between the vastus lateralis obliquus and vastus lateralis muscles.

Our study found significant increases in lateral patellar excursion at all levels of knee flexion, with the greatest differences seen in full extension, after lateral retinacular and capsular release compared with intact specimens. It is important to note that the reported percentages are differences in distances in response to a constant force rather than the force required to translate the patella a fixed distance. Prior studies have measured the change in force required to translate the patella a fixed difference, and thus, such percentages may not be directly comparable to those in our study. However, in contrast to previous reports, we found a significant increase in lateral displacement at all degrees of knee flexion after lateral retinacular and capsular release compared with intact specimens. Prior studies have noted significant differences only at lower degrees of flexion. ${ }^{12,14-16}$ In these studies, force was increased until lateral patellar translation reached $10 \mathrm{~mm}$, whereas in our study, a constant force was applied and translation was measured. Thus, the different force-displacement methodology used for data collection may be more sensitive in detecting subtle but significant changes at higher degrees of flexion. For example, in a more lax specimen, $10 \mathrm{~mm}$ of displacement may still be within the toe-in region of the load-versus-displacement curve in which a limited lateral retinacular and capsular release will have less effect than in a stiffer specimen, in which translating the patella $10 \mathrm{~mm}$ would result in a point in the linear region of the curve. Furthermore, with the specimens in full extension, we noted a $30 \%$ increase in lateral translation compared with the intact specimens with a constant $20-\mathrm{N}$ force, which is slightly higher than that reported in the previous literature. At $10^{\circ}$ to $90^{\circ}$ of flexion, increases in lateral translation were all between $6 \%$ and $9 \%$, which is similar to prior reports. These data expand on prior studies and emphasize that regardless of the methodology used to measure changes in lateral stability, lateral retinacular and capsular release destabilizes the patellofemoral joint. However, although we were able to achieve statistical significance in lateral displacement between various levels of knee flexion, this may not correlate to clinical

Table 3. Percentage Increase in Lateral Displacement in Lateral-Release Specimens Compared With Intact Specimens as Function of Knee Flexion

\begin{tabular}{lc}
\hline Flexion, $^{\circ}$ & $\begin{array}{c}\text { Mean Increase in } \\
\text { Lateral Displacement } \\
(95 \% \mathrm{CI}), \%\end{array}$ \\
\hline 0 & $32.07(23.73-36.42)$ \\
10 & $7.16(5.10-9.23)$ \\
20 & $8.27(5.65-10.89)$ \\
30 & $8.01(4.90-11.1)$ \\
45 & $6.54(3.29-9.79)$ \\
60 & $6.29(4.43-8.27)$ \\
90 & $8.07(4.9-11.23)$ \\
\hline CI, confidence interval. & \\
${ }^{*}$ Significant finding $(P<0.05)$. &
\end{tabular}


significance given the relatively small absolute differences between our results. It is difficult and beyond the scope of this study to postulate whether our results are clinically significant; however, this study does suggest that lateral patellar stability is impacted by capsular and retinacular release. Certainly, further clinical studies are needed to determine the true clinical effect of such releases.

\section{Limitations}

There are several limitations to this study, many of which are inherent to using cadaveric specimens as a model for patellofemoral disorders that commonly occur in younger individuals. First, patellofemoral instability is complex and multifactorial and is highly influenced by neuromuscular control and activation with various activities. Thus, although we attempted to replicate at least a part of such neuromuscular activation by loading the quadriceps, this was far from complete and is clearly a major limitation of our study. We assumed normal patellofemoral alignment despite not having whole-leg specimens, and thus, our results may not be applicable to patients with chronic patellar malalignment. Furthermore, we did not conduct any patellofemoral joint force measurements. In addition, although we attempted to perform a limited and targeted lateral release that simulated an arthroscopic technique reflective of a modern understanding of the lateral patellar restraints, the release was performed in an open fashion; thus, our results may not exactly translate to an arthroscopic technique.

The results of our cadaveric study have direct clinical implications for patients undergoing surgery for lateral patellar instability. The finding that limited lateral retinacular and capsular release results in increased lateral patellar instability at all degrees of knee flexion suggests that this procedure should be used with extreme caution when combined with other procedures for patellar instability. Similarly, even limited releases performed in an arthroscopic manner raise the same concerns. Further studies are needed to determine which repair or reconstruction methods are biomechanically adequate to treat patients who have undergone release and have resultant iatrogenic medial instability.

\section{Conclusions}

Lateral retinacular and capsular release results in significantly increased lateral patellar translation at all flexion angles compared with intact specimens. This finding suggests that the lateral retinaculum may function as a significant secondary restraint to lateral translation.

\section{References}

1. Alaia MJ, Cohn RM, Strauss EJ. Patellar instability. Bull Hosp Jt Dis (2013) 2014;72:6-17.

2. Krompinger JW, Fulkerson JP. Lateral retinacular release for intractable lateral retinacular pain. Clin Orthop Relat Res 1983;179:191-193.

3. Miller R, Bartlett J. Recurrent patella dislocation treated by closed lateral retinacular release. Aust $N$ Z J Surg 1993;63:200-202.

4. Schonholtz GJ, Zahn MG, Magee CM. Lateral retinacular release of the patella. Arthroscopy 1987;3:269-272.

5. Aglietti P, Pisaneschi A, Buzzi R, Gaudenzi A, Allegra M. Arthroscopic lateral release for patellar pain or instability. Arthroscopy 1989;5:176-183.

6. Arshi A, Cohen JR, Wang JC, Hame SL, McAllister DR, Jones KJ. Operative management of patellar instability in the United States. Orthop J Sports Med 2016;4: 2325967116662873.

7. Fabbriciani C, Panni SA, Delcogliano A. Role of arthroscopic lateral release in the treatment of patellofemoral disorders. Arthroscopy 1992;8:531-536.

8. Henry JH, Goletz TH, Williamson B. Lateral retinacular release in patellofemoral subluxation. Am J Sports Med 1986;14:121-129.

9. Larson R, Cabaud H, Slocum D, James S, Keenan T, Hutchinson T. The patellar compression syndrome: Surgical treatment by lateral retinacular release. Clin Orthop Relat Res 1978;134:158-167.

10. Tan S, Chua C, Doshi C, Wong K, Lim A, Hui J. The outcomes of isolated lateral release in patellofemoral instability: A systematic review and meta-analysis [published online May 25, 2019]. J Knee Surg, https://doi.org/ 10.1055/s-0039-1688961.

11. Fithian DC, Paxton EW, Post WR, Panni A, International Patellofemoral Study Group. Lateral retinacular release: A survey of the International Patellofemoral Study Group. Arthroscopy 2004;20:463-468.

12. Bedi H, Marzo J. The biomechanics of medial patellofemoral ligament repair followed by lateral retinacular release. Am J Sports Med 2010;38:1462-1467.

13. Song G-Y, Hong L, Zhang H, Zhang J, Li Y, Feng H. Iatrogenic medial patellar instability following lateral retinacular release of the knee joint. Knee Surg Sports Traumatol Arthrosc 2016;24:2825-2830.

14. Christoforakis J, Bull A, Strachan R, Shymkiw R, Senavongse W, Amis A. Effects of lateral retinacular release on the lateral stability of the patella. Knee Surg Sports Traumatol Arthrosc 2006;14:273-277.

15. Merican AM, Kondo E, Amis AA. The effect on patellofemoral joint stability of selective cutting of lateral retinacular and capsular structures. J Biomech 2009;42: 291-296.

16. Desio SM, Burks RT, Bachus KN. Soft tissue restraints to lateral patellar translation in the human knee. Am J Sports Med 1998;26:59-65.

17. Nomura E, Horiuchi Y, Kihara M. Medial patellofemoral ligament restraint in lateral patellar translation and reconstruction. Knee 2000;7:121-127. 
18. Hautamaa PV, Fithian DC, Kaufman KR, Daniel DM, Pohlmeyer AM. Medial soft tissue restraints in lateral patellar instability and repair. Clin Orthop Relat Res 1998;349:174-182.

19. Tanner SM, Garth WP, Soileau R, Lemons JE. A modified test for patellar instability: The biomechanical basis. Clin J Sport Med 2003;13:327-338.
20. Wang Q, Huang W, Cai D, Huang H. Biomechanical comparison of single- and double-bundle medial patellofemoral ligament reconstruction. J Orthop Surg Res 2017;12:29.

21. Shah KN, DeFroda SF, Ware J, Koruprolu SC, Owens BD. Lateral patellofemoral ligament: An anatomic study. Orthop J Sports Med 2017;5:2325967117741439. 